\title{
O controle da tuberculose: um desafio para a saúde pública
}

\author{
Tuberculosis' control: a challenge for the public health
}

\author{
Maria Rita Bertolozzi ${ }^{1}$, Renata Ferreira Takahashi², Paula Hino ${ }^{3}$, \\ Marcelo Litvoc ${ }^{4}$, Francisco Oscar de Siqueira França ${ }^{5}$
}

Bertolozzi MR, Takahashi RF, Hino P, Litvoc M, França FOS. O controle da tuberculose: um desafio para a saúde pública/ Tuberculosis' control: a challenge for the public health. Rev Med (São Paulo). 2014 abr.-jun.;93(2):83-9.

RESUMO: A Tuberculose persiste como problema de Saúde Pública. O perfil da doença mostra que incide nos grupos que apresentam vulnerabilidades, principalmente de ordem social, o que denota a sua associação com a determinação social do processo saúde-doença. Apresentam-se, ainda, aspectos sobre a epidemiologia, clínica, tratamento e ações de prevenção.

DESCRITORES: Tuberculose/prevenção \& controle; Tuberculose/terapia; Tuberculose/diagnóstico; Tuberculose/ epidemiologia; Tuberculose/mortalidade; Vulnerabilidade social; Medicina clínica; Mycobacterium tuberculosis; Saúde pública.

\begin{abstract}
Tuberculosis remains a public health problem. The disease profile shows that groups that present vulnerabilities, mainly social, are particularly affected, denoting its association with social determinants of the health-disease process. Aspects of the epidemiology, clinical presentation, treatment and prevention actions are also presented.
\end{abstract}

KEY WORDS: Tuberculosis/prevention \& control; Tuberculosis/ therapy; Tuberculosis/diagnosis; Tuberculosis/epidemiology; Tuberculosis/mortality; Social vulnerability; Clinical medicine; Mycobacterium tuberculosis; Public health.

\footnotetext{
1. Enfermeira. Professora Associada do Departamento de Enfermagem em Saúde Coletiva (ENS) da Escola de Enfermagem (EE) da Universidade de São Paulo (USP). E-mail: mrbertol@usp.br.

2. Enfermeira. Professora Associada do Departamento ENS da EEUSP. E-mail: rftakaha@usp.br.

3. Enfermeira. Pós-Doutorado na EEUSP. E-mail: paulahino@yahoo.com.br.

4. Médico-Assistente. Divisão de Clínica de Doenças Infecciosas e Parasitárias do Hospital das Clínicas da Faculdade de Medicina da Universidade de São Paulo. E-mail: malitvoc@uol.com.br.

5. Médico. Professor Associado do Departamento de Moléstias Infecciosas e Parasitárias da Faculdade de Medicina da Universidade de São Paulo - FMUSP. E-mail: fosfranca@gmail.com.br.

Endereço para correspondência: Av. Dr. Enéas de Carvalho Aguiar, 419. Cerqueira César. São Paulo, SP, Brasil. CEP: $05403-000$.
} 


\section{INTRODUÇÃO}

A Tuberculose (TB) é, atualmente, um dos mais importantes desafios a serem enfrentados no âmbito da saúde pública, sendo a segunda causa de morte, no mundo, do grupo de doenças infecto-contagiosas, após a AIDS. Aproximadamente um terço da população mundial encontra-se infectada pelo bacilo causador da doença e há cerca de 10 milhões de doentes/ano, com 1 a 2 milhões de mortes/ano ${ }^{1,2}$.

A Organização Mundial da Saúde (OMS) destaca o Brasil como um dos 22 países prioritários para o controle da doença. Estes países são responsáveis por cerca de 80\% da carga mundial desta enfermidade. Ainda que o Brasil apresente queda da incidência de cerca de $20,3 \%$ desde 2003, em 2013, foram registrados 71.230 novos casos de tuberculose, sendo 11.500 de retratamento. O número de óbitos apresenta-se em torno de $5.000^{3,4}$. A distribuição da TB no Brasil é bastante heterogênea, entre seus municípios e regiões ${ }^{4}$.

ATB é exemplo consagrado da Determinação Social do Processo Saúde-Doença ${ }^{5-10}$. Ao mesmo tempo em que é extensamente associada aos processos de desigualdades, causa repercussões sociais, ao acometer os grupos de maior vulnerabilidade e os segmentos populacionais economicamente ativos, gerando impacto na economia das sociedades e intenso sofrimento naqueles que a vivenciam ${ }^{11}$.

A enfermidade está profundamente relacionada a outras condições como o acesso restrito aos serviços públicos de saúde, a limitada adesão ao tratamento, o aumento do número de casos de tuberculose causados por bacilos com resistência crescente*, o comprometimento da imunidade celular, como ocorre nas neoplasias, nos pacientes que fazem uso de fármacos com atividade imunodepressora (lúpus, artrite reumatóide, transplantados de órgãos, etc.), nos desnutridos, nos obesos e nos idosos. Todas essas condições, entre outras, têm influenciado o quadro epidemiológico mundial da TB.

$\mathrm{O}$ controle da enfermidade tem como alvo central a detecção precoce e o tratamento adequado dos doentes. Assim, é fundamental que os profissionais de saúde estejam devidamente capacitados para reconhecer, o mais rapidamente possível, os sinais e sintomas da TB e que apoiem os doentes no processo de tratamento, visando diminuir a sua transmissão. Também é fundamental que atuem fortemente no fomento às ações de prevenção ${ }^{12}$.

\section{Qual é o agente etiológico que causa a Tuberculose?}

A TB é causada pelo complexo Mycobacterium tuberculosis (M. tuberculosis, M. bovis, M. microti, M. africanum, M. canetti). A bactéria foi identificada por Robert Koch, em 1882, e é conhecida por bacilo de Koch. É um bacilo aeróbio e álcool-ácido resistente (BAAR), que se multiplica lentamente.

\section{Como ocorre a transmissão da Tuberculose?}

A transmissão, principalmente respiratória, ocorre quando são inaladas as partículas, sob a forma de aerossóis, que contém o bacilo, e que se encontram no meio ambiente. Isto é verificado quando ocorre contato íntimo e prolongado da pessoa doente com aquelas que convivem no mesmo domicílio ou no trabalho e estas últimas são denominadas comunicantes ${ }^{13}$.

As formas clínicas podem ser classificadas em: pulmonar, extrapulmonar e miliar. A forma pulmonar é a principal forma em que ocorre a transmissão e, neste caso, o bacilo é encontrado no escarro.

A fala, o espirro e a tosse carreiam gotículas, que podem ser responsáveis pela transmissão da $\mathrm{TB}$, no caso da forma pulmonar. A doença é transmitida de pessoa a pessoa por gotículas expelidas pelos enfermos, através da fala, do espirro e da tosse, sendo que as que têm maior peso depositam-se no solo. As mais leves podem permanecer em suspensão, no ar, por diversas horas. Os bacilos que se depositam em roupas e em utensílios dificilmente se dispersam em aerossóis. Portanto, nesse caso, não são importantes na transmissão da doença.

\section{O que ocorre no organismo após a entrada do bacilo?}

Ao inalar as partículas infectantes (com o bacilo de Koch), desenvolve-se, no pulmão, o complexo primário pulmonar $^{14,15}$. Depois, há disseminação aos linfáticos regionais, seguida de disseminação linfohematogênica. $\mathrm{Na}$ grande maioria dos contatos, ou seja, aproximadamente em $95 \%$ das situações, ocorre infecção e evolução para latência. A minoria, 5\%, desenvolverá a doença na primoinfecção.

Quando se estabelece a resposta imunológica, o indivíduo que está infectado permanece com o bacilo viável e desenvolve hipersensibilidade, que pode ser verificada por meio da conversão da Prova Tuberculínica (PPD - Protein Purified Derivative - que, antigamente,

\footnotetext{
*Exemplificados por bacilos MDR (multidroga resistentes): bacilos que são simultaneamente resistentes à Rifampicina (R) e à Isoniazida (I) e cepas XDR ("extensivamente drogaresistentes"): que além de resistentes à R e a I, também apresentam resistência a alguma fluoroquinolona e a alguma droga injetável (amicacina, kanamicina ou capreomicina).
} 
era conhecida por teste de Mantoux). Após o contato prévio, poderá haver reativação endógena da infecção latente pelo M.tuberculosis, em aproximadamente 5 a 10\% dos portadores do bacilo. É importante destacar que, nas situações em que a imunidade celular está diminuída, a exemplo da senescência, síndrome metabólica, infecção pelo HIV/AIDS e outras situações citadas anteriormente, aumenta a probabilidade da evolução para a doença, o que impõe um desafio adicional ao controle da TB. A seguir são descritas formas clínicas de TB.

\section{A Forma Pulmonar}

Conforme mencionado anteriormente, a forma pulmonar é a mais frequente e a mais contagiosa. Indivíduos portadores do bacilo, quando não tratados, podem infectar aproximadamente 10 a 15 pessoas/ano ${ }^{14,15}$.

A forma primária pulmonar é mais comum no caso das crianças e desenvolve-se, em geral, em curto período de tempo, após a formação do complexo primário (em até dois anos). A febre em geral é baixa, há sudorese noturna e falta de apetite.

A forma pós-primária pode ocorrer em qualquer idade, mas é mais comum nos adultos jovens. A principal característica é a tosse, que pode ser seca ou produtiva. $\mathrm{O}$ escarro pode apresentar sangue. A febre ao entardecer, em geral atinge o limite de $38,5^{\circ} \mathrm{C}$. Há sudorese noturna, falta de apetite e a pessoa pode apresentar "face fatigada" e emagrecimento.

\section{A TB miliar}

Corresponde ao comprometimento de, pelo menos, dois órgãos não vizinhos, simultaneamente, sendo mais frequente em menores de 5 anos, não vacinados com BCG; em idosos e em imunodeprimidos. Os principais sinais e sintomas são: emagrecimento, febre e astenia ${ }^{13}$.

\section{A TB extra-pulmonar}

As formas extra-pulmonares de TB envolvem órgãos e sistemas, e a gravidade varia com o local e a intensidade do acometimento ${ }^{15}$.

a. Pleural: o sintoma mais importante é a dor pleurítica acompanhada de tosse seca e desconforto respiratório. É a mais comum e ocorre em jovens.

b. Ganglionar: pode ocorrer febre e os gânglios são palpáveis e indolores. É mais comum nos adolescentes e adultos jovens e também pode ser encontrada nos pacientes HIV soropositivos.

c. Ósteo-articular: nos adultos, o local mais acometido é a coluna toraco-lombar e o sintoma mais comum é a lombalgia crônica.

d. Abdominal: o paciente apresenta alterações intestinais, geralmente diarréia crônica; febre e fadiga. No trato gastrointestinal, o local mais frequentemente acometido, é o íleo terminal. Também pode acometer o peritônio e gânglios abdominais, que podem evoluir para ascite.

e. Neuro-Tuberculose: é sempre muito grave, com importante risco de morte. Em geral, os pacientes apresentam cefaléia crônica, fadiga, febre, alterações comportamentais, crises convulsivas, dentre outras manifestações. A apresentação mais comum é a meningite subaguda.

f. Gênito-Urinária: neste caso, podem ser encontrados sintomas urinários, como disúria, hematúria, polaciúria e dor supra-púbica. Deve sempre ser pesquisada em pacientes com suposta Infecção do Trato Urinário (ITU), mas com uroculturas persistentemente negativas.

g. Ocular: pode apresentar-se como uma conjuntivite ou acometer as pálpebras e o aparelho lacrimal. Os sinais e sintomas mais comuns são: vermelhidão ocular, diminuição da acuidade visual e sensação de corpo estranho.

h. Cutânea: lesões ulceradas na pele, com evolução crônica, que podem fistulizar.

i. Pericárdica: geralmente apresenta dor torácica, tosse seca e dispnéia.

\section{Que exames podem ajudar no diagnóstico da Tuber-} culose?

O principal exame é o microbiológico e envolve a pesquisa do bacilo e a cultura do material. Além do escarro, qualquer tecido pode ser submetido à baciloscopia, que é a procura direta do bacilo, e à cultura ${ }^{16}$.

Para a baciloscopia são necessárias duas amostras de escarro: a primeira deverá ser coletada ainda na primeira consulta do doente. A segunda amostra deverá ser coletada na manhã do dia seguinte, logo ao despertar e encaminhada à unidade básica de saúde (UBS). Ao contrário da baciloscopia, cujo resultado é muito rápido (em horas), a cultura costuma apresentar resultado somente a partir de 30 dias. 
O volume ideal de escarro é de cerca de 5 a $10 \mathrm{ml} \mathrm{e}$ a melhor amostra é a que decorre do esforço de tosse. As Unidades Básicas de Saúde (UBS), em geral, disponibilizam os frascos para a coleta de material. Os frascos devem ser descartáveis e ter tampa de rosca.

O Raio-X é importante exame para o diagnóstico de todas as formas de TB, mas não é suficiente, pois aproximadamente $15 \%$ dos casos de TB pulmonar não apresentam alterações nesse exame. Nos pacientes com baciloscopia positiva, o Raio-X tem, principalmente, a função de excluir doença pulmonar associada, como, por exemplo, no caso de câncer pulmonar, além de avaliar a extensão do comprometimento pulmonar ${ }^{16}$.

$$
\mathrm{O} \quad \mathrm{T} \text { e s t e }
$$

Tuberculínico é usado para evidenciar o contato prévio com o bacilo, mas não denota a doença em atividade. É importante considerar o importante número de resultados falsonegativos e positivos do teste. O Teste deve ser aplicado na face anterior do antebraço esquerdo, na UBS, na dose de $0,1 \mathrm{ml}$ (2 UT de tuberculina). Após 48 a 72 horas deverá ser realizada a leitura do maior diâmetro transverso da enduração (e não o eritema). A idade, o tamanho da enduração, o risco de adoecimento por TB deverão orientar a interpretação do Teste Tuberculínico ${ }^{16}$.

Atualmente, já há métodos imunológicos para a detecção de anticorpos na TB. Entretanto, seu alto custo, além da dificuldade da padronização das técnicas e, principalmente, sua limitada especificidade em detectar os pacientes com a doença, ainda não viabilizaram seu uso extensivo no Brasil. Técnicas como a Reação em Cadeia de Polimerase (PCR) e outros métodos de Biologia Molecular, que apresentam maior rapidez nos resultados, estão sendo progressivamente utilizados, para abreviar o tempo entre a coleta do material e o diagnóstico.

\section{Tratamento da Tuberculose}

O tratamento tem duas fases ${ }^{16,17,18}$ :

- Fase intensiva: Rifampicina, Isoniazida, Pirazinamida, Etambutol: nos dois primeiros meses. Nesta fase deve ser utilizada a forma "Quatro em Um" (Coxcip 4): ou seja, quatro medicamentos em um só comprimido. O número de comprimidos varia segundo o peso do paciente.

- Fase de manutenção: Rifampicina, Isoniazida por quatro meses.

O comprimido deve ser tomado em jejum, uma hora antes do café da manhã ou 2 horas após. O tratamento é inteiramente gratuito em todo o território nacional.

Há esquemas especiais nos casos de intolerância aos medicamentos, ou no caso de hepatotoxicidade, de hipersensibilidade ou de resistência às drogas.

O tratamento supervisionado (TS) é uma estratégia muito importante para aumentar a adesão ao tratamento $^{19}$. Consiste na observação do paciente ingerindo os medicamentos, regularmente, por um profissional de saúde ou por uma pessoa próxima ao doente e que se responsabilize pelo procedimento. Esta estratégia deve se constituir em um momento de troca entre o profissional de saúde e o paciente e possibilita oportunidade para que sejam abordadas eventuais dúvidas e dificuldades. Portanto, não deve resumir-se à simples observação da ingestão do medicamento pela pessoa enferma ${ }^{11,20}$.

Mesmo após tanto tempo decorrido da descoberta do bacilo e da existência de tratamento eficaz, é importante 
considerar que ainda há estigma e preconceito em relação à $\mathrm{TB}$, além de ser enfermidade de cunho eminentemente social. Tais fatos, além do extenso período de tratamento, dos possíveis efeitos colaterais, assim como as condições de vulnerabilidade em que se encontram a maior parte das pessoas acometidas, demandam aos trabalhadores da saúde dialogar com os pacientes, para a identificação de necessidades de saúde, pois estas podem dificultar a adesão ao tratamento ${ }^{11}$.

\section{A Tuberculose}

multidrogaresistente (TBMDR) e a Tuberculose extra-resistente (XDR-TB)

Referem-se à resistência às drogas utilizadas para o tratamento. Segundo o Ministério da Saúde ${ }^{16}$, a TBMR é o: "Caso de tuberculose, com diagnóstico por cultura e identificação de M. tuberculosis e teste de sensibilidade com resistência à rifampicina e isoniazida”.

Em 2007, surgiu outro desafio no controle da TB, quando foram encontradas, na África do Sul, cepas "extensivamente resistentes" (XDR-TB, extensively drug-resistant tuberculosis). Essas cepas apresentam resistência à rifampicina, à isoniazida, a qualquer fluorquinolona e a qualquer droga injetável: (a amicacina, a capreomicina e a canamicina) $)^{21,22}$.

A resistência decorre, principalmente, do tratamento irregular, o que coloca em destaque a importância do rápido diagnóstico e instalação do tratamento ${ }^{23}$. Também é fundamental a adesão ao tratamento, ou seja, a regularidade da tomada da medicação no período e nas doses prescritas pelo profissional médico.

A resistência é evidenciada quando o paciente apresenta baciloscopia positiva, mesmo após o $4^{\circ}$ mês de tratamento.

\section{A Tuberculose e a AIDS}

É sempre necessário pensar em TB quando a pessoa apresenta AIDS e pensar em AIDS, quando a pessoa apresenta TB, dada a associação entre ambas as enfermidades. É importante destacar que é muito frequente descobrir que o paciente é HIV positivo durante o diagnóstico de TB. Portanto, é sempre relevante a abordagem integral do paciente com garantia de acesso ao teste HIV e tratamento antiretroviral, quando for o caso ${ }^{16}$.

No caso de risco para a infecção, como por exemplo, no caso de recém-nascidos que vivem em ambiente com pacientes bacilíferos, deve ser realizada a Profilaxia Primária, que consiste na administração de uma das drogas, a Isoniazida, antes mesmo da BCG.

No caso de pessoas que apresentem mudança da prova Tuberculínica, tornando-se reatores, ou no caso de alguma condição que cause imunodepressão, ou no caso de pessoas que convivam ou trabalhem, ou seja, que tenham contato mais íntimo e prolongado com bacilíferos indica-se a Profilaxia Secundária, que consiste na administração de Isoniazida.

\section{Ações recomendadas durante o Tratamento}

O estímulo à adesão ao tratamento é uma das ações fundamentais no controle da TB e deve iniciar-se tão logo o paciente receba o diagnóstico ${ }^{16}$. Neste momento, é importante:

1. Esclarecer sobre a doença: como é transmitida, a importância de identificar o caso índice e os comunicantes. O esclarecimento ajuda a mitigar o preconceito em relação à enfermidade;

2. Esclarecer sobre a importância do tratamento: diário e as doses corretas. Identificar as condições concretas que o doente tem para o tratamento. Exemplos: o paciente tem condições para alimentar-se? Tem suporte financeiro para ir à UBS? Tem acesso à unidade de saúde sempre quando necessitar? Há alguma barreira geográfica ou física que o impeça ou que seja obstáculo para que vá à unidade de saúde? O paciente tem condições físicas para dirigir-se à unidade de saúde ou o TS deve ser realizado no domicílio? A decisão sobre o TS deverá sempre ser objeto de discussão aberta e decisão conjunta entre o paciente (ou alguém por ele responsável) e o profissional de saúde. Quando o TS for realizado no domicílio, é sempre necessário manter o sigilo;

3. Verificar se o paciente requer apoio por parte de outras pessoas da família, ou outros, vizinhos, amigos, para o tratamento;

4. Esclarecer sobre eventuais efeitos colaterais das drogas, sobre a necessidade de não interrupção do tratamento, e de informar aos profissionais de saúde sobre sinais e sintomas que podem ser decorrentes da medicação;

5. Orientar sobre uso de lenço para tossir e espirrar. Não varrer o solo, mas utilizar pano úmido e deixar o ambiente ventilado e com o máximo de entrada de sol. 


\section{A Vigilância Epidemiológica é sempre fundamental}

A prevenção da TB é uma das atividades que compõem a Vigilância Epidemiológica. A vacina BCG, administrada sempre que possível nos primeiros 15 dias após o nascimento, por via intradérmica, tem proteção que varia e não permanece por toda a vida ${ }^{16}$. O calendário de vacinação deverá sempre ser seguido, segundo recomendação do Ministério da Saúde.

A vacinação para o grupo infantil tem como objetivo principal, evitar apresentações graves da doença, como a tuberculose miliar e a neurotuberculose.

Todos os casos de TB devem ser notificados às instâncias que compõem a Vigilância Epidemiológica ${ }^{16}$. Também é necessário identificar os comunicantes domiciliares, no trabalho e na escola. As pessoas consideradas como sintomáticos respiratórios (tosse que persiste por mais de 21 dias) deverão ser submetidas à baciloscopia de escarro $^{24}$.

O profissional de saúde deverá também monitorar os pacientes e verificar os faltosos às consultas médica e de enfermagem, além do TS ${ }^{19}$. É sempre necessário identificar os motivos que levam ao não comparecimento à UBS e buscar fazer com que o paciente encontre-se acolhido na unidade de saúde, ou seja, garantir-lhe acessibilidade no sentido mais amplo do termo, ou seja: acesso geográfico, ao atendimento sempre que necessário, sem obstáculos na relação com os profissionais de saúde.

Após a notificação da enfermidade, o paciente deverá receber visita domiciliária, que é importante ação da equipe de saúde e tem a finalidade de obter elementos sobre as condições de vida e de subsistência da família, além das possibilidades para o enfrentamento da doença. É na visita domiciliária que é possível, na maior parte das ocasiões, entender como a enfermidade poderá ser enfrentada com o apoio dos familiares e/ou de vizinhos.

Além da primeira visita para a identificação do caso índice e dos comunicantes, as visitas domiciliárias devem ser realizadas periodicamente, como forma de comprometer a unidade de saúde em relação às necessidades e situação de saúde do doente e seus familiares.

É na visita domiciliária que serão identificados os comunicantes, e é nesse espaço que o profissional de saúde pode informar os familiares a respeito da doença e deixar emergir dúvidas a respeito de sua transmissão e tratamento.

Conforme mencionado anteriormente, quando o tratamento tem início e uma vez que seja realizado corretamente, o risco de transmissão, em geral, declina a partir do $15^{\circ}$ dia. Essa informação é fundamental ao paciente e seus familiares, porque pode apoiar o envolvimento destes em relação ao doente e modificar eventuais atitudes de isolamento, decorrentes de preconceito e estigma.

Também é importante apontar que o seguimento do tratamento faz com que o doente apresente, em geral, logo ao final do primeiro mês de tratamento, melhora importante de suas condições gerais. Muitos pacientes deixam de tomar a medicação exatamente nesse período. Portanto, é importante esclarecê-lo, e a seus familiares, a respeito, e enfatizar a necessidade de prosseguir o tratamento até sua conclusão.

Durante a visita domiciliária há uma série de informações que a unidade de saúde deve prover às pessoas em geral e, particularmente aquelas que apresentem a TB, ou seus familiares, como por exemplo:

- cobrir a boca e o nariz ao tossir e/ou espirrar;

- manter a moradia ventilada e aproveitar o sol;

- a moradia deve ser limpa com pano úmido para evitar a disseminação de aerossóis;

- buscar conversar para diminuir o preconceito e o estigma em relação à doença;

- o tabaco deve ser evitado por todos os familiares;

- estimular ações positivas que acabem por convergir no enfrentamento conjunto da doença.

\section{CONCLUSÃO}

A TB ainda é importante causa de morbi-mortalidade, o que exige a ação dos vários profissionais de saúde, melhorando a gestão dos serviços e a assistência.

O agente etiológico é o Mycobacterium tuberculosis, bacilo álcool ácido-resistente (BAAR). A via respiratória é a principal forma de transmissão, a partir de partículas aerossolisadas de pacientes. O teste anti-HIV deve ser oferecido a todos os pacientes com TB - doença, independentemente da forma clínica ou idade.

A TB é doença de notificação compulsória. As ações de Vigilância Epidemiológica incluem, além da prevenção, a notificação compulsória, a identificação de comunicantes e o monitoramento do tratamento do paciente. O TS é estratégia para aumentar a adesão ao paciente.

O médico e os demais profissionais da saúde são essenciais para a garantia da adesão ao tratamento, devendo acolher as necessidades apresentadas pelos doentes que, muitas vezes, podem não imediatamente apresentar-se como necessidades de cunho clínico, biológico, mas que evidenciam necessidades sociais, com decorrências para a manifestação e o enfrentamento da enfermidade.

Dado que a TB é enfermidade de cunho eminentemente social, a sua persistência decorre das inadequadas condições sociais e das iniquidades. Assim, o seu controle pressupõe o entendimento da doença a partir de 
uma perspectiva ampla, que envolve a determinação social e a sua superação decorrerá da superação das desigualdades sociais. O profissional de saúde deve compreender que o adoecimento se deve a condições de vida, definidas pela inserção social, que define as vulnerabilidades, tanto

\section{REFERÊNCIAS}

1. Lönnroth K, Castro KG, Chacaya JM, Chauhan LS, Floyd K, Glaziou P, Raviglione M. Tuberculosis control and elimination 2010-50: cure, care, and social development. Lancet. 2010;375:1814-29. doi: 10.1016/S0140-6736(10)60483-7.

2. World Health Organization. Global tuberculosis control: surveillance, planning, financing. Geneva: WHO Report; 2012.

3. World Health Organization. Global tuberculosis report 2013. Available from: http://www.who.int/tb/publications/ global_report/gtbr13_annex_2_country_profiles.pdf

4. Brasil. Ministério da Saúde. Portal da Saúde. Disponível em: http://www.brasil.gov.br/Saude/2014.

5. Breilh J. Precisamos ter um novo viver, com taxas de crescimento menos agressivas, mas com mais qualidade [entrevista]. Rev Poli: Saude Educ Trab. 2011;20(4):11-3. Disponível em: http:// www.epsjv.fiocruz.br/upload/EdicoesRevistaPoli/R22.pdf.

6. Paim JS. Atenção primária à saúde: uma receita para todas as estações? Saúde Debate. 2012;36(94):343-47. Disponível em: http://dx.doi.org/10.1590/S0103-11042012000300004.

7. Lopes R, Tocantins FR. Promoção da saúde e a educação crítica. Interface Comunic Saude Educ. 2012;16(40):23546. Disponível em: http://www.scielo.br/pdf/icse/v16n40/ aop1312.

8. Santos LRCS, Silva TPC. A utopia da reforma sanitária brasileira em um discurso no Congresso da ABRASCO 2009. Saúde Debate. 2013;37(97):210-8. Disponível em: http:// www.scielo.br/pdf/sdeb/v37n97/v37n97a03.pdf.

9. Benatar SR. Some oxygen, please, for anoxic poverty alleviation strategies. Int J Tuberc Lung Dis. 2011;15(11):1425. doi: 10.5588/ijtld.11.0321.

10. Lönnroth K, Weil D, Raviglione M, Jaramillo E. Tuberculosis and poverty: what is being done. Int J Tuberc Lung Dis. 2011;15(4):431-2. doi: 10.5588/ijtld.10.0654.

11. Bertolozzi MR. A adesão ao Programa de Controle da Tuberculose no Distrito Sanitário do Butantã, São Paulo [tese]. São Paulo: Faculdade de Saúde Pública da Universidade de São Paulo; 1998.

12. Barreira D, Grangeiro A. Avaliação das estratégias de controle da tuberculose no Brasil. Rev Saúde Pública. 2007;41(Supl. 1): 4-8. Disponível em: http://www.scielo.br/pdf/rsp/v41s1/ apresentacao.pdf.

13. Litvoc M, França FOS, Bertolozzi MR. Tuberculose. São Paulo: Guanabara Koogan; 2014.

14. Melo FAF, et al. Tuberculose. In: Focaccia R, Veronesi R. Tratado de infectologia. 4a ed. São Paulo: Atheneu; 2009.

15. Mandell GL, Bennett JE, Dolin R. Mandell's principles and pessoais como coletivas. Assim, esta abordagem mais ampla do processo saúde doença, permitirá a compreensão da situação de saúde do paciente, do território onde vive e das possibilidades para a superação dos diversos contextos de vulnerabilidade. practice of infectious diseases. New York: Elsevier/Churchill Livingstone; 2005.

16. Brasil. Ministério da Saúde. Secretaria de Vigilância em Saúde. Departamento de Vigilância Epidemiológica. Manual de recomendações para o controle da tuberculose no Brasil. Brasília; 2011. Disponível em: http://www.cve.saude.sp.gov. $\mathrm{br} / \mathrm{htm} / \mathrm{TB} / \mathrm{mat}$ tec/manuais/MS11_Manual_Recom.pdf.

17. Brasil. Ministério da Saúde. Secretaria de Vigilância em Saúde. Departamento de Vigilância Epidemiológica. Programa Nacional de Controle da Tuberculose. Nota técnica sobre as mudanças no tratamento da tuberculose no Brasil para adultos e adolescentes. Brasília; 2009. Disponível em: link:http:// www.hc.ufpr.br/files/nota_tecnica_sobre_as_mudancas_no_ tratamento_da_tuberculose_no_brasil.pdf.

18. São Paulo. Secretaria de Estado da Saúde. Coordenadoria de Controle de Doenças. Centro de Vigilância Epidemiológica Prof. Alexandre Vranjac. Divisão de Tuberculose. Mudanças no tratamento da tuberculose. Rev Saúde Pública. 2010;44(1):197-9. Disponível em: http://www.scielosp.org/ $\mathrm{pdf} / \mathrm{rsp} / \mathrm{v} 44 \mathrm{n} 1 / 22$.pdf.

19. Brasil. Ministério da Saúde. Secretaria de Vigilância em Saúde. Departamento de Vigilância Epidemiológica. Tratamento diretamente observado (TDO) da tuberculose na atenção básica. Brasília; 2011. Disponível em: http://bvsms.saude. gov.br/bvs/publicacoes/tratamento_diretamente_observado_ tuberculose.pdf.

20. Terra MF, Bertolozzi MR. Does directly observed treatment ("DOTS") contribute to tuberculosis treatment compliance? Rev Latino-am Enfermagem. 2008;16(4):659-64. http://dx.doi. org/10.1590/S0104-11692008000400002

21. Jassal M, Bishai WR. Extensively drug-resistant tuberculosis. Lancet Infect Dis. 2009;9:19-30. doi: 10.1016/S14733099(08)70260-3.

22. Migliori GB, Centis R, Lange C, Richardson MD, Sotgiu G. Emerging epidemic of drug-resistant tuberculosis in Europe, Russia, China, South America and Asia: current status and global perspectives. Curr Opin Pulm Med. 2010;16:171-9. doi: 10.1097/MCP.0b013e328337573e.

23. Dalcolmo MP, Andrade MK, Picon PD. Tuberculose multiresistente no Brasil: histórico e medidas de controle. Rev Saude Pública. 2007;41(Supl.1):34-42. Disponível em: http:// www.scielosp.org/pdf/rsp/v41s1/6570.pdf.

24. Sociedade Brasileira de Pneumologia e Tisiologia. Comissão de Tuberculose/ Grupo de Trabalho das Diretrizes para Tuberculose. III Diretrizes para tuberculose da Sociedade Brasileira de Pneumologia e Tisiologia. J Bras Pneumol. 2009;35(10):1018-48. http://dx.doi.org/10.1590/S180637132009001000011 . 